\title{
Modificaciones Morfológicas del Pie Luego de Ocho Semanas de Entrenamiento de Carrera a Pie Descalzo
}

\author{
Foot Morphological Adaptations Developed After Eight Weeks of Barefoot Running Training
}

Sánchez-Ramírez ${ }^{1}$, Celso \& Alegre, Luis M².

SÁNCHEZ-RAMíREZ, C. \& ALEGRE, L. M. Modificaciones morfológicas del pie luego de ocho semanas de entrenamiento de carrera a pie descalzo. Int. J. Morphol., 37(3):1111-1117, 2019.

RESUMEN: La carrera descalza ha sido ampliamente estudiada últimamente, pero poco se sabe de cambios anatómicos que produce en el pie. El objetivo de este estudio fue determinar estos cambios producidos de forma aguda y a largo plazo en sujetos que entrenan a pie descalzo. 29 sujetos divididos en dos grupos: Barefoot (BFGr) ( $n=17)$ y Shod (SGr) ( $n=12)$, realizaron un protocolo de efecto agudo consistente en correr durante $20 \mathrm{~min}$ sobre treadmill a $3.1 \mathrm{~m} / \mathrm{s}$ y un protocolo de entrenamiento de 8 semanas de carrera de resistencia con aumento progresivo de volumen. BFGr realizó todas las sesiones a pie descalzo. Al finalizar cada protocolo se midió Foot Length (FL), Forefoot Width (FW), Hindfoot Width (HW), Navicular Height (NH), Arch Index (AI) y Maximum Surface (MS). Se efectuó ANOVA 3x2 para comprobar los efectos que el tiempo y la condición de calzado produjo. La condición de calzado produjo efectos significativos sobre todas las variables (FL: $\mathrm{p}=0.000, \eta^{2} \mathrm{p}=0.997 ; \mathrm{FW}: \mathrm{p}=0.000, \eta^{2} \mathrm{p}=0,997 ; \mathrm{HW}: \mathrm{p}=0.000, \eta^{2} \mathrm{p}=0$,994; NH: $\mathrm{p}=0.000, \eta^{2} \mathrm{p}=0.953$; AI: $\left.\mathrm{p}=0.000, \eta^{2} \mathrm{p}=0.898 ; \mathrm{MS}: \mathrm{p}=0.000, \eta^{2} \mathrm{p}=0.983\right)$ y el factor tiempo sobre AI $\left(\mathrm{p}=0.012, \eta^{2} \mathrm{p}=0.152\right)$ y MS $(\mathrm{p}=0.000$, $\left.\eta^{2} \mathrm{p}=0.259\right)$. Hubo interacción significativa Tiempo x Condición en FW ( $\left.\mathrm{p}=0.036, \eta^{2} \mathrm{p}=0.116\right)$ y NH $\left(\mathrm{p}=0.019, \eta^{2} \mathrm{p}=0.143\right)$. MS aumentó luego del protocolo de efecto agudo $(\mathrm{p}=0.000)$ y a largo plazo $(\mathrm{p}=0.001)$ en $\mathrm{BFGr}$. El porcentaje de sujetos con pie normal aumentó en BFGr y con pie plano aumentó en SGr. El entrenamiento de carrera a pie descalzo produce efectos, principalmente luego de un período de adaptación de 8 semanas, tendientes a un aumento armónico en la superficie de apoyo plantar con tendencia a una disminución relativa del área del mediopié, lo cual se tradujo en una disminución de sujetos con arco plantar de tipología plana.

PALABRAS ClAVE: Descalzo; Corriendo; Pie; Pie plano; Morfología; Presión plantar.

\section{INTRODUCCIÓN}

El pie humano supone el punto de contacto del cuerpo con el suelo, transformándose en un elemento de la anatomía humana que permite transmitir fuerzas durante la bipedestación y las acciones de locomoción (Miralles \& Miralles, 2005), gracias a estructuras altamente especializadas como el Arco Plantar Longitudinal Medial (MLFA). La morfología del pie además, puede describirse según sus dimensiones externas, evaluadas generalmente a través de antropometría directa, y cuya utilidad se basa principalmente en la capacidad de poder determinar las medidas apropiadas que debe tener el calzado que una población emplee, debido a que la morfología del pie presenta amplia variabilidad entre sujetos, según sexo, etnia, estado de gestación y factores ambientales tales como el uso de calzado y la práctica de actividad deportiva (Kulthanan et al., 2004).
Durante los últimos años, gracias a la idea impulsada principalmente por Bramble \& Lieberman (2004) y con un enfoque evolutivo, se ha despertado el interés por estudiar el comportamiento mecánico del pie durante la carrera cuando se encuentra desprovisto de calzado o con calzado minimalista, comúnmente conocido como Barefoot Running (BFR). Los mencionados autores se basan en el hecho que el autralophitecus afarensis logró erguirse hace cerca de 2.2 millones de años, siendo la carrera de resistencia, una práctica que catalizó este proceso, realizado claramente, sin la mediación de calzado (Campbell et al., 2006). Este antecedente, toma fuerza debido a que no ha sido posible disminuir la incidencia de lesiones a pesar de la popularidad que tiene entre los deportistas el uso de calzado deportivo especializado, manteniéndose la tasa de lesiones anuales en valo-

\footnotetext{
${ }^{1}$ Laboratorio de Ciencias de la Actividad Física - Facultad de Ciencias Médicas - Universidad de Santiago de Chile, Santiago, Chile.

${ }^{2}$ Universidad de Castilla-La Mancha, Grupo de Investigación GENUD Toledo, Toledo, España. CIBER de Fragilidad y Envejecimiento Saludable, España. Estudio financiado por la Dirección de Investigación Científica y Tecnológica (Dicyt) de la Universidad de Santiago de Chile, Proyecto Dicyt Regular código 021887SR
} 
res que oscilan entre el $30 \%$ y $75 \%$ de los corredores (Daoud et al., 2012). Desde este enfoque teórico, se han desarrollado ya numerosos estudios orientados principalmente a caracterizar los cambios agudos y crónicos que se producen en los sujetos que han comenzado a correr descalzos o con calzado minimalista (Azevedo et al., 2016; Au et al., 2017). Estos estudios eso sí, se han enfocado fundamentalmente en variables de índole biomecánica, tales como cinética y cinemática articular, y no en aspectos anatómicos del pie (Hollander et al., 2017). Por esto, el objetivo de esta investigación fue determinar los cambios morfológicos del pie y del MLFA que se producen inmediatamente después de una sesión de carrera a pie descalzo, y determinar las adaptaciones a largo plazo que sufre la morfología del pie y del MLFA luego de 8 semanas de entrenamiento bajo esta misma condición.

\section{MATERIAL Y MÉTODO}

Sujetos: La muestra se conformó inicialmente por 42 sujetos, pero sólo 29 completaron el protocolo experimental. Dos de los sujetos que no completaron el protocolo se retiraron debido a abrasiones de la piel que no sanaron a tiempo como para completar el período de entrenamiento. Veinticuatro hombres y 5 mujeres, estudiantes de ciencias de la actividad física, de 20,0 $\pm 1,5$ años; 71,1 $\pm 10,5 \mathrm{~kg}$ de peso corporal; $1,72 \pm 0,07 \mathrm{~m}$ deestatura y un índice de masa corporal (BMI) de $24,1 \pm 2,5 \mathrm{~kg} \cdot \mathrm{m}^{-2}$. Los sujetos no tenían experiencia corriendo a pie descalzo y corrían de forma regular entre 5 y $10 \mathrm{~km}$ a la semana. Ninguno de los participantes había sufrido lesión de tobillo y/o pie durante los últimos seis meses previos al inicio del estudio. Los sujetos participaron de forma voluntaria en esta investigación, firmando el formulario de consentimiento informado redactado y aprobado según las directrices del Comité de Ética Institucional de la Universidad de Santiago de Chile (Informe Ético N¹84-2018).
Procedimiento protocolo efecto agudo. Se aplicó un cuestionario de elaboración propia de salud podálica, postural e historial deportivo (Sánchez et al., 2017) y se definió su pie dominante (Lake et al., 2011). Se conformaron dos grupos: Barefoot (BFGr) de $n=17$ y Shod (SGr) de $n=12$, los cuales no presentaron diferencias significativas en las variables de descripción. Antes de efectuar las mediciones, cada sujeto guardó reposo durante 10 min en posición decúbito supino (Jiménez-Ormeño et al., 2011). Luego, se llevaron a cabo las mediciones diagnósticas (Baseline) consistentes en la medición de peso y estatura, la evaluación antropométrica del pie y luego la obtención de presiones plantares. Inmediatamente después, cada participante corrió en una cinta rodante (Technogym, mod. Excite Run 500 , Italia) durante 20 minutos a $3,1 \mathrm{~m} \cdot \mathrm{s}^{-1}$ con una pendiente de $5 \%$. Esta velocidad se ha usado más frecuentemente en los estudios de cinemática de carrera y de evaluación de presiones plantares relacionados con BFR (Warne et al., 2017). Los sujetos de SGr corrieron con su calzado deportivo convencional y los sujetos de BFGr corrieron a pies descalzos. Una vez finalizado este intento, volvieron a ser evaluados para determinar el efecto agudo que produce el correr descalzo (Post 20 min Run).

Procedimiento protocolo efecto largo plazo. Durante las 8 semanas siguientes a Post 20 min Run, los sujetos cumplieron un protocolo de entrenamiento consistente en la práctica de carrera continua, a velocidad autoseleccionada, durante 24 sesiones, distribuidas en 3 sesiones semanales, durante 8 semanas. SGr lo realizó con su calzado deportivo convencional y el BFGr lo llevó a cabo absolutamente descalzo. El volumen de entrenamiento se midió en minutos y se incrementó de forma ondulante, tal como se indica en la Tabla I. Una vez finalizado el protocolo y luego de tener 48 a 72 horas de descanso, se evaluó a los sujetos por tercera vez (Post 8 week Training). Se realizaron de esta manera, tres evaluaciones (Fig. 1).

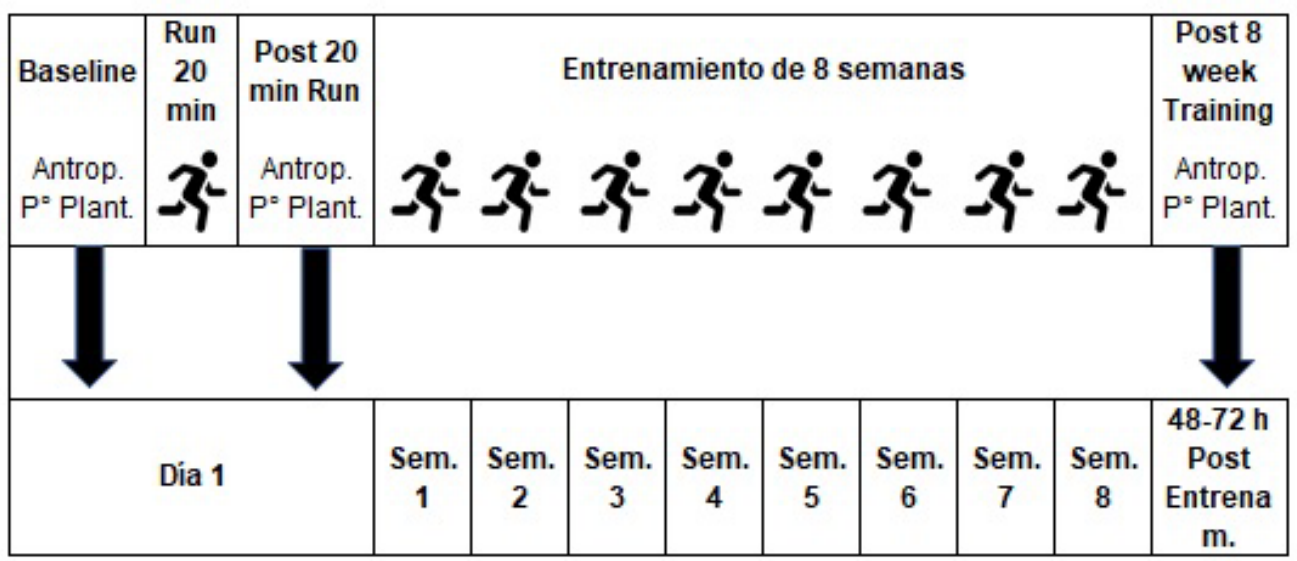

Fig. 1. Esquema temporal del protocolo de evaluación y de entrenamiento. Antrop. = Medición antropométrica; $\mathrm{P}^{\circ}$ Plant. $=$ Presiones plantares 
Tabla I. Determinación del volumen de entrenamiento del protocolo experimental a lo largo de las 8 semanas y 24 sesiones de entrenamiento.

\begin{tabular}{|c|c|c|c|c|c|c|c|c|c|c|c|c|}
\hline & \multicolumn{3}{|c|}{ Semana 1} & \multicolumn{3}{|c|}{ Semana 2} & \multicolumn{3}{|c|}{ Semana 3} & \multicolumn{3}{|c|}{ Semana 4} \\
\hline Sesión $n^{\circ}$ & 1 & 2 & 3 & 4 & 5 & 6 & 7 & 8 & 9 & 10 & 11 & 12 \\
\hline Minutos & 5 & 7 & 5 & 8 & 10 & 8 & 15 & 10 & 10 & 20 & 10 & 15 \\
\hline & \multicolumn{3}{|c|}{ Semana 5} & \multicolumn{3}{|c|}{ Semana 6} & \multicolumn{3}{|c|}{ Semana 7} & \multicolumn{3}{|c|}{ Semana 8} \\
\hline S esión $n^{\circ}$ & 13 & 14 & 15 & 16 & 17 & 18 & 19 & 20 & 21 & 22 & 23 & 2 \\
\hline Minutos & 25 & 15 & 25 & 30 & 25 & 30 & 35 & 30 & 35 & 35 & 40 & 3 \\
\hline
\end{tabular}

Mediciones: Se efectuó la medición antropométrica, consistente en la obtención de la longitud del pie (FL), ancho del antepié (FW), ancho del retropié (HW) y altura navicular (NH) empleando un pie de metro análogo de $300 \mathrm{~mm}$ (Veto, E5002009, Chile), según protocolo especificado por Sánchez et al.

Para la obtención de la morfología de la huella plantar, se empleó baropodoscopio Presscam V4 (Sidas ${ }^{\odot}$, Francia), con 1600 sensores de captación. Los sujetos se ubicaron sobre la plataforma en bipedestación, con el peso de su cuerpo repartido de manera homogénea en ambos pies descalzos. Una vez estabilizada la posición, se obtuvo la impresión de las huellas plantares, adquiridas del promedio de $10 \mathrm{~s}$ a una frecuencia de $100 \mathrm{~Hz}$. De esta medición se obtuvo la Superficie Máxima (MS) del apoyo plantar, expresada en $\mathrm{cm}^{2}$. De la impresión obtenida desde el baropodoscopio, y mediante análisis en software AreaCalc (Elvira et al., 2008), se calculó el Índice del Arco de Cavanagh y Rodgers (AI) (Cavanagh \& Rodgers, 1986), que determina la tipología de MLFA (cavo, plano o normal).

Confiabilidad de las mediciones de antropometría y de análisis de la huella plantar. Con el objetivo de analizar la confiabilidad intra examinador e interexaminador de la técnica de medición antropométrica y del análisis de la huella plantar, se llevó a cabo un estudio piloto donde se evaluó a 11 sujetos por 2 evaluadores distintos. Las mediciones se repitieron una semana después por ambos evaluadores. Se calculó el coeficiente de correlación intraclase para la consistencia de las mediciones intra examinador e inter examinador.

Análisis estadístico. Se consideró el pie dominante para todos los análisis. Se utilizó estadística descriptiva y prueba t para la comparación inicial de ambos grupos. Se llevó a cabo un análisis de varianzas de medidas repetidas (ANOVA) 3×2 para establecer efectos e interacciones que el factor Tiempo produce sobre cada una de las variables (Baseline/Post 20 min Run/Post 8 week Training), así como los efectos e interacciones que el factor uso de calzado deportivo produce (SGr/BFGr). Si era aplicable, se realizó análisis Post hoc con corrección de Bonferroni. Se calculó homocedasticidad me- diante test de Lèvene y esfericidad en base a la prueba de Mauchly. El tamaño del efecto, expresado como eta cuadrado parcial $\left(\eta^{2} \mathrm{p}\right)$, indicó el porcentaje de varianza en cada uno de los efectos y su error asociado que se explica por ese efecto. Se determinó $\mathrm{p} \leq 0,05$ como valor de significancia estadística. Todos los análisis se realizaron empleando el programa de análisis estadístico SPSS (IBM, V23.0 Inc., Chicago, IL, USA).

\section{RESULTADOS}

Las pruebas de confiabilidad, medidos mediante el coeficiente de correlación intraclase (ICC) entregaron elevados valores de confiabilidad intra examinador $(>0,88)$ e inter examinador $(>0,82)$. El error típico de la media osciló entre 1,2 y $5,2 \mathrm{~mm}$ para medidas antropométricas, entre 0,01 y 0,02 para AI, y entre 4,2 y $4,9 \mathrm{~cm}^{2}$ para la superficie de apoyo plantar.

A modo de establecer igualdad de condiciones, se compararon los resultados obtenidos por ambos grupos en Baseline, en cada una de las variables estudiadas, demostrándose igualdad estadística (Tabla II).

El factor condición de calzado (Shod Group/Barefoot Group) produjo efectos significativos en todas las variables y el factor tiempo o momento de evaluación (Baseline/Post 20 min Run/Post 8 week Training) produjo efectos significativos en casi todas las variables.

En MS, se encontraron efectos significativos del factor tiempo $\left(\mathrm{F}=9,440 ; \mathrm{p}=0,000 ; \eta^{2} \mathrm{p}=0,259\right)$ y del factor condición de calzado $\left(\mathrm{F}=1520,238 ; \mathrm{p}=0,000 ; \eta^{2} \mathrm{p}=0,983\right)$ por separado. El análisis post hoc arrojó diferencias significativas entre Baseline y Post 8 week Training $(\mathrm{p}=0,001)$ y entre Post 20 min Run y Post 8 week Training $(\mathrm{p}=0,005)$ en ambos grupos. Asimismo, se observó que dentro del Barefoot Group hubo diferencias significativas entre Baseline y Post 8 week Training marcada por un aumento del $9,1 \%(\mathrm{p}=0,001)$ y entre Post 20 min Run y Post 8 week Training con un aumento del $14,82 \%(\mathrm{p}=0,000)$. 
Hubo interacción significativa entre los efectos del factor tiempo (Baseline/Post 20 min Run/Post 8 week Training) y del factor condición de calzado (Shod Group/ Barefoot Group) en las variables FW ( $\mathrm{F}=3,529 ; \mathrm{p}=0,036$; $\left.\eta^{2} \mathrm{p}=0,116\right)$ y $\mathrm{NH}\left(\mathrm{F}=4,515 ; \mathrm{p}=0,019 ; \eta^{2} \mathrm{p}=0,143\right)$ (Tabla III). $\mathrm{FW}$ presenta diferencias entre grupos $(\mathrm{F}=7872,614 ; \mathrm{p}=0,000$; $\eta^{2} \mathrm{p}=0$,997) donde eta parcial al cuadrado indica que el 99,7 $\%$ de la varianza fue explicada por el factor condición. El análisis post hoc arrojó que hubo diferencias significativas entre ambos grupos en Post 8 week Training $(p=0,049)$, siendo los sujetos de Shod Group quienes presentaron antepié un $4,68 \%$ más anchos.

$\mathrm{NH}$ también presentó diferencias entre grupos $\left(\mathrm{F}=541,434 ; \mathrm{p}=0,000 ; \eta^{2} \mathrm{p}=0,953\right)$. El análisis post hoc indica que la altura navicular fue un 10,69\% mayor en Post 8 week Training que en Post 20 min Run en Shod Group.

AI también se vio influenciada significativamente por el factor tiempo $\left(\mathrm{F}=4,826 ; \mathrm{p}=0,012 ; \eta^{2} \mathrm{p}=0,152\right)$ y por el factor condición $\left(\mathrm{F}=238,714 ; \mathrm{p}=0,000 ; \eta^{2} \mathrm{p}=0,898\right)$ por separa- do. El análisis post hoc indica que hubo diferencias significativas entre los valores obtenidos en ambos grupos en Baseline $\mathrm{y}$ en Post 8 week Training $(\mathrm{p}=0,026)$, representadas por un aumento del $12,30 \%$.

FL fue influenciada significativamente por el efecto del factor condición ( $\left.\mathrm{F}=8858,223 ; \mathrm{p}=0,000 ; \eta^{2} \mathrm{p}=0,997\right)$. El análisis post hoc indica que los sujetos del Barefoot Group experimentaron una disminución del 0,43\% en la longitud de pie entre Baseline y Post 8 week Training $(\mathrm{p}=0,047)$. Asimismo, el factor condición muestra efecto significativo sobre la variable HW ( $\left.\mathrm{F}=4253,327 ; \mathrm{p}=0,000 ; \eta^{2} \mathrm{p}=0,994\right)$ no encontrando diferencias significativas en el análisis post hoc.

La Tabla IV muestra los resultados obtenidos del análisis del MLFA. La distribución del tipo de pie fue desigual en ambos grupos. En Baseline el SGr presentó mayoría de pies cavos $(41,7 \%)$, mientras que BFGr presentó mayoría de pies planos $(52,9 \%)$. En Post 20 min Run, fue posible observar que SGr mostró una tendencia al aplanamiento del MLFA, al evidenciarse un aumento de los pies planos $(41.7 \%)$ y dis-

Tabla II. Comparación de ambos grupos en cada una de las variables estudiadas durante Baseline.

\begin{tabular}{lccc}
\hline & Shod Group & Barefoot Group & $\mathrm{p}$ \\
\hline Edad (años) & $\mathrm{N}=12$ & $\mathrm{~N}=17$ & 0,12 \\
Peso $(\mathrm{kg})$ & $20,5 \pm 1,5$ & $19,7 \pm 1,4$ & 0,14 \\
Estatura (m) & $74,5 \pm 10,7$ & $68,7 \pm 9,9$ & 0,55 \\
Índice de Masa Corporal $\left(\mathrm{kg} \cdot \mathrm{m}^{-2}\right)$ & $1,72 \pm 0,06$ & $1,71 \pm 0,08$ & 0,06 \\
Longitud (mm) & $25,1 \pm 2,6$ & $23,3 \pm 2,3$ & 0,79 \\
Ancho de antepié $(\mathrm{mm})$ & $256,1 \pm 15,6$ & $257,8 \pm 17,7$ & 0,27 \\
Ancho de retropié $(\mathrm{mm})$ & $103,3 \pm 5,5$ & $100,7 \pm 6,7$ & 0,51 \\
Altura navicular $(\mathrm{mm})$ & $68,8 \pm 4,6$ & $62,4 \pm 6,4$ & 0,75 \\
Índice del Arco & $34,8 \pm 8,7$ & $35,0 \pm 8,5$ & 0,73 \\
Superficie máxima $\left(\mathrm{cm}^{2}\right)$ & $0,20 \pm 0,09$ & $0,21 \pm 0,09$ & 0,25 \\
\hline
\end{tabular}

Tabla III. Resultados obtenidos por ambos grupos en los tres momentos de evaluación.

\begin{tabular}{lcccccc}
\hline & \multicolumn{3}{c}{ Shod Group } & \multicolumn{3}{c}{ Barefoot Group } \\
\cline { 2 - 6 } & baseline & Post 20 Min Run & Post 8 Week & Baseline & Post 20 Min & Post 8 Week \\
\hline Longitud (mm) & $256,1 \pm 15,6$ & $256,0 \pm 15,9$ & $256,4 \pm 15,7$ & $257,8 \pm 17,7$ & $257,5 \pm 17,5$ & $256,7 \pm 17,8^{\mathrm{a}}$ \\
Ancho antepié $(\mathrm{mm})$ & $103,3 \pm 5,5$ & $103,2 \pm 6,1$ & $105,2 \pm 6,7^{*}$ & $100,7 \pm 6,7$ & $101,4 \pm 7,2$ & $100,5 \pm 5,6^{*}$ \\
Ancho retropié $(\mathrm{mm})$ & $63,8 \pm 4,6$ & $64,5 \pm 4,7$ & $64,8 \pm 3,9$ & $62,4 \pm 6,4$ & $63,2 \pm 6,0$ & $61,8 \pm 5,2$ \\
Altura navicular $(\mathrm{mm})$ & $34,8 \pm 8,7$ & $33,5 \pm 7,8$ & $37,1 \pm 8,1+$ & $35,0 \pm 8,5$ & $34,2 \pm 8,2$ & $32,9 \pm 8,5$ \\
Índice del Arco & $0,20 \pm 0,09$ & $0,21 \pm 0,09$ & $0,24 \pm 0,06^{\mathrm{f}}$ & $0,21 \pm 0,09$ & $0,21 \pm 0,08$ & $0,22 \pm 0,06^{\mathrm{f}}$ \\
Superficie máxima $\left(\mathrm{cm}^{2}\right)$ & $91,8 \pm 18,2$ & $98,7 \pm 19,1$ & $101,4 \pm 14,2$ & $84,5 \pm 15,3$ & $87,2 \pm 14,8$ & $100,1 \pm 12,8^{\mathrm{bc}}$ \\
\hline
\end{tabular}

a Longitud en Barefoot Group Post 8 Week Training fue menor que Baseline.

b Superficie máxima en Barefoot Group Post 8 Week Training fue mayor que Baseline.

c Superficie máxima en Barefoot Group Post 8 Week Training fue mayor que Post 20 min Running.

* Interacción significativa entre el factor tiempo y el factor condición de calzado. Ancho de antepié en Shod Group Post 8 Week Training fue mayor que Ancho de antepié en Barefoot Group Post 8 Week Training (post hoc).

†Interacción significativa entre el factor tiempo y el factor condición de calzado. Altura navicular en Shod Group Post 8 Week Training fue mayor que Post 20 min Running (post hoc).

f Índice del arco en Post 8 Week Training fue mayor que Post 20 min Running en ambos grupos. 
Tabla IV. Distribución de la tipología de pie según MLFA por cada grupo en cada uno de los momentos de evaluación.

\begin{tabular}{|c|c|c|c|c|c|c|}
\hline & \multicolumn{2}{|c|}{ Baseline } & \multicolumn{2}{|c|}{ Post 20 Min Run } & \multicolumn{2}{|c|}{ Post 8 Week } \\
\hline & $\mathrm{n}$ & $\%$ & $\mathrm{n}$ & $\%$ & $\mathrm{n}$ & $\%$ \\
\hline \multicolumn{7}{|c|}{ Shod Group } \\
\hline Plano & 4 & 33,3 & 5 & 41,7 & 6 & 50,0 \\
\hline Normal & 3 & 25,0 & 3 & 25,0 & 4 & 33,3 \\
\hline Cavo & 5 & 41,7 & 4 & 33,3 & 2 & 16,7 \\
\hline \multicolumn{7}{|c|}{ Barefoot Group } \\
\hline Plano & 9 & 52,9 & 8 & 47,1 & 6 & 35,3 \\
\hline Normal & 3 & 17,7 & 3 & 17,7 & 6 & 35,3 \\
\hline Cavo & 5 & 29,4 & 6 & 35,3 & 5 & 29,4 \\
\hline
\end{tabular}

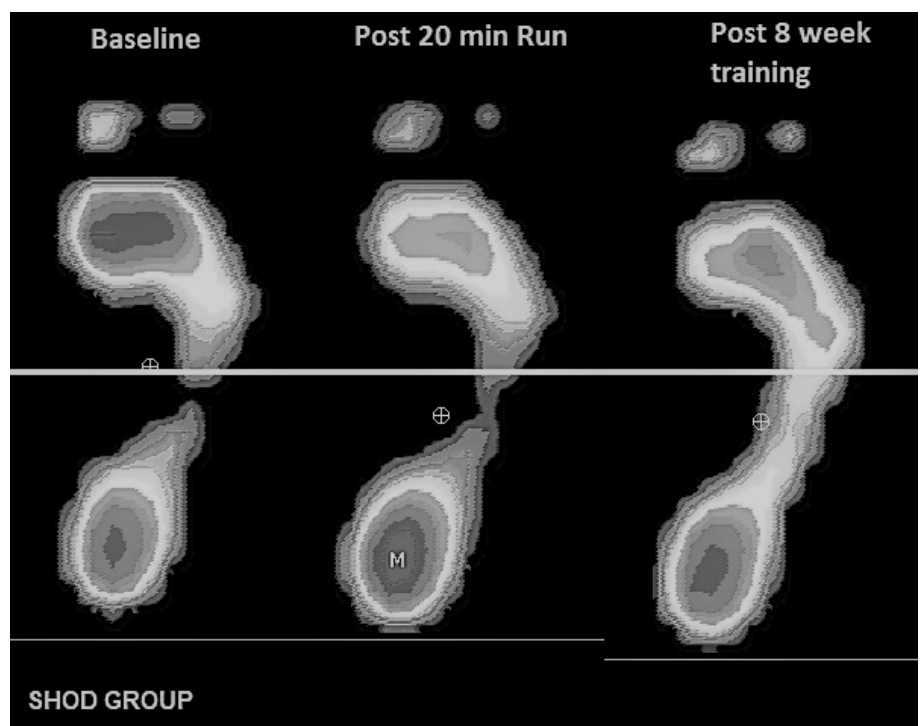

Fig. 2. Ejemplo de modificaciones típicas agudas y crónicas de la huella plantar de un sujeto de Shod Group. Es posible observar un aplanamiento de la bóveda plantar y un aumento de la Superficie de contacto del apoyo plantar de Baseline $\left(\mathrm{AI}=0,20 ; \mathrm{MS}=120 \mathrm{~cm}^{2}\right)$ a Post 8 week training ( $\mathrm{AI}=0,26 ; \mathrm{MS}=122 \mathrm{~cm}^{2}$ ).

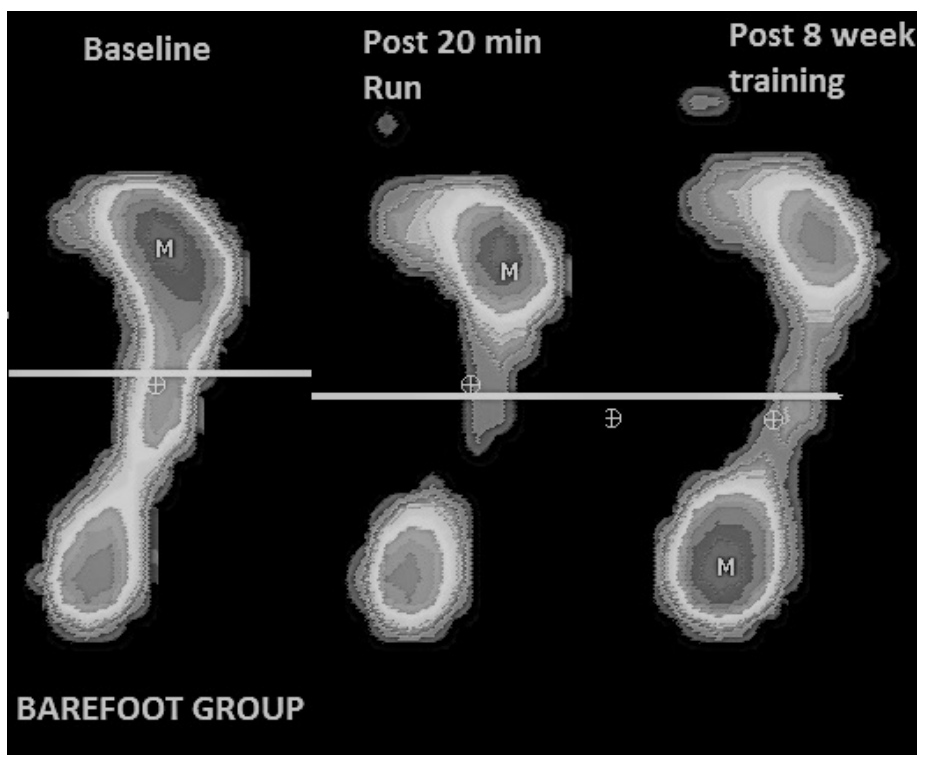

minución de los pies cavos $(33,3 \%)$. BFGr por el contrario, evidenció una ligera disminución en el porcentaje de pies planos $(47,1 \%)$ y pequeño aumento de porcentaje de pies cavos $(35,3 \%)$. En Post 8 week Training, es posible de ver que SGr continuó con la tendencia hacia el aplanamiento del MLFA, aumentando el porcentaje de frecuencia de pies planos, de un tercio a la mitad, así como a una disminución en el porcentaje de sujetos con pies cavos $(16,7 \%)$. En el BFGr, el protocolo de entrenamiento de 24 sesiones provocó la disminución en el número de sujetos con pies planos $(35,3$ $\%$ y aumentó el número de sujetos con pies normales $(35,3 \%)$. Se mantuvo el porcentaje de pies cavos registrados en Baseline. Las Figuras 2 y 3 ilustran los cambios experimentados por la morfología de la huella plantar en los tres momentos de evaluación por un sujeto perteneciente a SGr y un sujeto perteneciente a BFGr.

\section{DISCUSIÓN}

El objetivo de este estudio del tipo longitudinal fue determinar los cambios agudos y a largo plazo que sufre la morfología del pie de sujetos que comienzan a correr desprovistos de calzado. Al respecto, es pertinente indicar que, a la fecha, son pocos los estudios experimentales longitudinales en materia de carrera minimalista, y menos aún, los que se centran en variables morfológicas, aseveración respaldada por el reciente estudio de Hollander et al. Los hallazgos más notorios son los relacionados con el efecto alcanzado luego de la práctica de 24 sesiones de carrera con aumento progresivo del volumen. En efecto agudo en cambio, el pie de los sujetos prácticamente no sufrió modificaciones en su morfología, a pesar de que corrieron durante $20 \mathrm{~min}$ a $3,1 \mathrm{~m} \cdot \mathrm{s}^{-1}$.

La variable MS fue la variable que experimentó los mayores cambios orientados hacia el incremento, en los distintos momentos de evaluación y en ambas condiciones de uso de calzado, siendo este último factor el que produjo los efectos más importantes. Cabe destacar que fue la única variable que presentó modificaciones significativas en efecto agudo (Post $20 \mathrm{~min}$ Run).

Fig. 3. Ejemplo de modificaciones típicas agudas de la huella plantar en un sujeto de Barefoot Group. Es posible observar una elevación de la bóveda plantar y un aumento de la Superficie de contacto del apoyo plantar de Baseline $\left(\mathrm{AI}=0,27 ; \mathrm{MS}=89 \mathrm{~cm}^{2}\right)$ a Post 8 week training $(\mathrm{AI}=0,22$; MS $=97 \mathrm{~cm}^{2}$ ). 
En una revisión que analizó las modificaciones agudas que se producen luego de correr largas distancias, Kim et al. (2018) no encontraron evidencia de cambios significativos en la superficie de apoyo plantar luego de correr un maratón con calzado deportivo. Delgado-Abellán et al. (2012) por su parte, encontraron que luego de una sesión de carrera de $2500 \mathrm{~m}$ de volumen a una velocidad menor que la realizada en este estudio, se produjo un aumento del 2,7 \% de esta variable, y que luego de una sesión de carrera de 30 min aumentó un 3,3\%, con significancia estadística, resultados que no se lograron demostrar en la presente investigación, probablemente debido a que se emplearon distintas superficies de carrera (Nigg et al., 1995).

Los mayores aumentos de MS se observaron en post 8 week training. Este efecto podría contrastarse con los estudios de diseño longitudinal de Miller et al. (2014) y Johnson et al. (2016) que midieron el área de sección transversal (CSA) de músculos intrínsecos del pie antes y después de un entrenamiento a pie descalzo. Los primeros autores encuentran que el músculo abductor del dedo pequeño y el flexor corto de los dedos aumentan su volumen luego de 12 semanas de adaptación al BFR en el grupo que entrenó con calzado minimalista, al igual que el segundo estudio que reporta que en sujetos que entrenaron 10 semanas con calzado minimalista, CSA del músculo abductor del hallux aumentó un 10,6\%. Este crecimiento muscular podría relacionarse con un aumento relativo de la superficie de apoyo plantar, principalmente en la zona del mediopié, lugar donde se halla el vientre de los músculos mencionados. Asimismo, Delgado-Abellán et al. encontraron que el área del mediopié aumentaba entre un 3,5\% y 9,1\% luego de una sesión de carrera de 2500 m y de 30 min respectivamente. Esta hipótesis, no obstante, no se comprueba en este estudio, ya que en el BFGr al parecer este aumento de superficie de apoyo plantar se desarrolló más probablemente en las zonas del antepié y retropié, debido a que disminuyó el número de sujetos con pie plano.

En esta misma línea, Mei et al. (2015) estudiaron a sujetos divididos según patrón de apoyo plantar, quienes corrieron a $3,0 \mathrm{~m} \cdot \mathrm{s}^{-1}$ en condiciones de calzado y descalzo, encontrando que los sujetos que habitualmente se mueven descalzos tienen menor área de superficie de apoyo en la zona medial del mediopié lo cual indica un MLFA de tipología cava, resultado que coincide con los cambios descritos en la distribución de tipología de pie, en donde los sujetos que corrieron descalzos tuvieron tendencia a modificar su arco plantar hacia un tipo cavo, en Post 20 min Run y Post 8 week Training.

Shu et al. (2015) estudiaron a dos poblaciones de sujetos que normalmente corren a pie descalzo (indios) con otro grupo de corredores calzados (chinos), obteniendo como resultado que las mujeres del primer grupo tienen pies más largos y antepiés más anchos, lo cual podría ser una adaptación natural. D'AoÛt et al. (2009) reportan resultados similares, en donde sujetos que caminan descalzos también muestran pies más anchos que quienes utilizan calzado, pero poseen pies relativamente más cortos. En la presente investigación se registró un aumento de FW en SGr y una disminución de FL en BFGr luego de las 8 semanas de entrenamiento, resultados que coinciden parcialmente con los estudios citados. Cabe destacar que estos tuvieron un diseño transversal y no aplicaron un protocolo de entrenamiento descalzo. Al parecer, y según lo que plantean Shu et al., D'AoÛt et al., Mei et al. y Kadambande et al. (2006), el tiempo de adaptación a la locomoción descalza es el factor que hace la diferencia, ya que las adaptaciones morfológicas en personas que nunca han usado calzado son distintas a las que sufren quienes siempre han usado calzado y que en una edad adulta pasan a prescindir de esta prenda. De todas maneras, los cambios registrados por FL en esta investigación deben ser tomados sólo a modo de referencia ya que la variación encontrada se traduce en una media de 1,1 mm de diferencia, valor que se encuentra dentro del error estándar de la media.

De esta investigación de tipo experimental, fue posible concluir que la condición descalza durante la práctica de carrera produce efectos, principalmente luego de un período de adaptación de 8 semanas, tendientes a producir modificaciones morfológicas específicas en el pie, consistentes en un aumento armónico en la superficie de apoyo plantar con tendencia a una disminución relativa del área del mediopié, lo cual se tradujo en una disminución de sujetos con MLFA de tipología plana.

Debido a la exigua existencia de estudios de este tipo, se sugiere realizar más investigaciones del tipo experimental, evaluando otros indicadores de la morfología del pie, para poder profundizar más en la temática.

SÁNCHEZ-RAMÍREZ, C. \& ALEGRE, L. M. Foot morphological adaptations developed after eight weeks of barefoot running training. Int. J. Morphol., 37(3):1111-1117, 2019.

SUMMARY: Barefoot running has been studied extensively recently, but little is known of the anatomical changes that take place in the foot. The objective of this study was to determine the acute and chronic changes that are produced in subjects who train barefoot. 29 subjects divided into two groups, Barefoot $(\mathrm{BFGr})(\mathrm{n}=17)$ and Shod $(\mathrm{SGr})(\mathrm{n}=12)$, followed an acute effect protocol which consisted in running during $20 \mathrm{~min}$ on a treadmill at $3.1 \mathrm{~m} / \mathrm{s}$, and an eight-week long chronic effect protocol covering eight weeks of resistance running with progressively increasing 
volume. BFGr did all the sessions barefoot. At the end of each protocol Foot Length (FL), Forefoot Width (FW), Hindfoot Width (HW), Navicular Height (NH), Arch Index (AI), and Maximum Surface (MS) were measured. ANOVA $3 \times 2$ was run to verify the effects caused by time and the shod condition produced. Shod/ Unshod factor caused significant effects on all the variables (FL: $\mathrm{p}=0.000, \eta^{2} \mathrm{p}=0.997 ; \mathrm{FW}: \mathrm{p}=0.000, \eta^{2} \mathrm{p}=0,997 ; \mathrm{HW}: \mathrm{p}=0.000, \eta^{2} \mathrm{p}=$ 0,994; NH: $p=0.000, \eta^{2} \mathrm{p}=0.953$; AI: $\mathrm{p}=0.000, \eta^{2} \mathrm{p}=0.898$; MS: $\left.\mathrm{p}=0.000, \eta^{2} \mathrm{p}=0.983\right)$, and the time factor on AI $(\mathrm{p}=0.012$, $\left.\eta^{2} \mathrm{p}=0.152\right)$ and MS $\left(\mathrm{p}=0.000, \eta^{2} \mathrm{p}=0.259\right)$. There was a significant Time $x$ Condition interaction in FW $\left(p=0.036, \eta^{2} p=0.116\right)$ and $\mathrm{NH}$ $\left(\mathrm{p}=0.019, \eta^{2} \mathrm{p}=0.143\right)$. MS increased after the acute $(\mathrm{p}=0.000)$ and the chronic $(\mathrm{p}=0.001)$ protocols in the BFGr. The percentage of subjects with normal feet increased in the BFGr and that with flat feet increased in SGr. Barefoot running training causes effects, mainly after an adaptation period of eight weeks, tending to a harmonic surface increase on the support plantar area, with a relative decrease of the midfoot area, which meant a decrease of subjects with plantar arch of flat type.

KEY WORDS: Barefoot; Running; Foot; Flatfoot; Morphology; Plantar Pressure.

\section{REFERENCIAS BIBLIOGRÁFICAS}

Au, I. P. H.; Lau, F. O. Y.; An, W. W.; Zhang, J. H.; Chen, T. L. \& Cheung, R. T. H. Immediate and short-term biomechanical adaptation of habitual barefoot runners who start shod running. J. Sports Sci., 36(4):451$5,2017$.

Azevedo, A. P.; Mezêncio, B.; Amadio, A. C. \& Serrão, J. C. 16 Weeks of Progressive Barefoot Running Training Changes Impact Force and Muscle Activation in Habitual Shod Runners. PLoS One, 11(12):e0167234, 2016.

Bramble, D. M. \& Lieberman, D. E. Endurance running and the evolution of Homo. Nature, 432(7015):345-52, 2004.

Campbell, R.; Lieberman, D. E. \& Scott, J. W. Why are our toes so tiny? Walking, running and the evolution of a short forefoot in the genus Homo. Integr. Comp. Biol., 212:713-21, 2006.

Cavanagh, P. R. \& Rodgers, M. M. The arch index: a useful measure from footprints. J. Biomech., 20(5):547-51, 1987.

D'aout, K.; Pataky, T. C.; De Clercq, D. \& Aerts, P. The effects of habitual footwear use: foot shape and function in native barefoot walkers. Footwear Sci., 1(2):81-94, 2009.

Daoud, A. I.; Geissler, G. J.; Wang, F.; Saretsky, J.; Daoud, Y. A. \& Lieberman, D. E. Foot strike and injury rates in endurance runners: a retrospective study. Med.Sci. Sports Exerc., 44(7):1325-34, 2012.

Delgado-Abellán, L.; Aguado, X.; Jiménez-Ormeño, E.; Mecerreyes, L. \& Alegre, L. M. Efectos del ejercicio continuo e intermitente sobre la huella plantar. Arch. Med. Deporte, 29(148):601-8, 2012.

Elvira, J. L.; Vera-García, F. J. \& Meana, M. Subtalar joint kinematic correlations with footprint arch index in race walkers. J. Sports Med. Phys. Fitness, 48(2):225-34, 2008.

Hollander, K.; de Villiers, J. E.; Sehner, S.; Wegscheider, K.; Braumann, K. M.; Venter, R. \& Zech, A. Growing-up (habitually) barefoot influences the development of foot and arch morphology in children and adolescents. Sci.Rep., 7(1):8079, 2017.

Jiménez-Ormeño, E.; Aguado, X.; Delgado-Abellan, L.; Mecerreyes, L. \& Alegre, L. M. Changes in footprint with resistance exercise. Int. J. Sports. Med., 32(8):623-8, 2011.

Johnson, A. W.; Myrer, J. W.; Mitchell, U. H.; Hunter, I. \& Ridge, S. T. The effects of a transition to minimalist shoe running on intrinsic foot muscle size. Int. J. Sports Med., 37(2):154-8, 2016.

Kadambande, S.; Khurana, A.; Debnath, U.; Bansal, M. \& Hariharan, K. Comparative anthropometric analysis of shod and unshod feet. Foot, 16(4):188-91, 2006.

Kim, H. K.; Mirjalili, S. A. \& Fernandez J. Gait kinetics, kinematics, spatiotemporal and foot plantar pressure alteration in response to longdistance running: Systematic review. Hum. Mov. Sci., 57:342-56, 2018

Kulthanan, T.; Techakampuch, S. \& Bed, N. D. A study of footprints in athletes and non-athletic people. J. Med. Assoc. Thai., 87(7):788-93, 2004.

Lake, J. P.; Lauder, M. A. \& Smith, N. A. Does side dominance affect the symmetry of barbell end kinematics during lower-body resistance exercise? J. Strength Cond. Res., 25(3):872-8, 2011.

Mei, Q.; Fernández, J.; Fu, W.; Feng, N. \& Gu, Y. A comparative biomechanical analysis of habitually unshod and shod runners based on a foot morphological difference. Hum. Mov. Sci., 42:38-53, 2015.

Miller, E. E.; Whitcome, K. K.; Lieberman, D. E.; Norton, H. L. \& Dyer, R. E. The effect of minimal shoes on arch structure and intrinsic foot muscle strength. J. Sport Health Sci., 3(2):74-85, 2014.

Miralles, R. \& Miralles, I. Biomecánica Clínica de los Tejidos y las Articulaciones del Aparato Locomotor. Barcelona, Masson, 2005.

Nigg, B. M.; De Boer, R. W. \& Fisher, V. A kinematic comparison of overground and treadmill running. Med. Sci. Sports Exerc., 27(1):98$105,1995$.

Sánchez, C.; Alarcón, E. \& Morales, H. Morphofunctional foot characteristics of chilean university athletes in ten sports disciplines. Int. J. Morphol., 35(4):1403-8, 2017.

Shu, Y.; Mei, Q.; Fernandez, J.; Li, Z.; Feng, N. \& Gu, Y. Foot morphological difference between habitually shod and unshod runners. PLoS One, 10(7): $\mathrm{e} 0131385,2015$.

Warne, J. P.; Smyth, B. P.; Fagan, J. O.; Hone, M. E.; Richter, C.; Nevill, A. M.; Moran, K. A. \& Warrington, G. D. Kinetic changes during a sixweek minimal footwear and gait-retraining intervention in runners. $J$. Sports Sci., 35(15):1538-46, 2017.

Dirección para correspondencia:

Celso Sánchez-Ramírez

Laboratorio de Ciencias de la Actividad Física

Facultad de Ciencias Médicas

Universidad de Santiago de Chile

Santiago

CHILE

Email: celso.sanchez@usach.cl

Recibido : 14-02-2019

Aceptado : 30-04-2019 